\title{
I mpacto do Projeto Armazém das Artes no Bem-estar dos Participantes
}

\section{I mpact of Armazém das Artes Project on Participants' Well- being}

\section{I mpacto del Proyecto Armazém das Artes en el Bienestar de los Participantes}

\author{
Luciana Nacif Pinheiro* \\ Universidade Salgado de Oliveira - UNIVERSO, Niterói, Rio de Janeiro, Brasil \\ Maria Cristina Ferreira** \\ Universidade Salgado de Oliveira - UNIVERSO, Niterói, Rio de Janeiro, Brasil \\ Luciana Mourão*** \\ Universidade Salgado de Oliveira - UNIVERSO, Niterói, Rio de Janeiro, Brasil
}

\begin{abstract}
RESUMO
O bem-estar no trabalho tem recebido destaque na literatura de psicologia organizacional. Sendo assim, investigou-se, neste artigo, o impacto do projeto Armazém das Artes no bem-estar de seus participantes. A amostra de 97 funcionários de uma universidade federal, de ambos os sexos, foi dividida em um grupo experimental $(\mathrm{N}=48)$ e em um grupo controle $(\mathrm{N}=$ 49). O grupo experimental participou de oito sessões semanais, nas quais foram ensinadas diversas técnicas de cartonagem, enquanto o grupo controle não recebeu nenhum tipo de intervenção. Ambos os grupos responderam a instrumentos destinados a avaliar seus níveis de bem-estar, ao início da primeira sessão (pré-teste) e ao final da última sessão (pósteste). As análises da variância (ANOVA) mistas 2 (grupo) X 2 (tempo), com medidas repetidas no fator tempo e medidas independentes no fator grupos, evidenciaram que os escores de comprometimento organizacional afetivo e de afetos positivos dirigidos ao trabalho foram significativamente maiores ao final da intervenção, enquanto os de exaustão emocional foram significativamente menores ao final da intervenção. Concluiu-se que o projeto mostrou-se eficaz no sentido de contribuir para a melhoria do bemestar de seus participantes.
\end{abstract}

Palavras-chave: avaliação, programas, bem-estar, atividades artísticas.

\section{ABSTRACT}

Well-being at work have been highlighted in the organizational psychology literature. The impact of the Armazém das Artes project on the well-being of its participants was investigated. The sample had included 97 employees from a federal university. The sample included men and women, divided in an experimental group $(\mathrm{N}=48)$ and in a control group $(\mathrm{N}=49)$. The 
experimental group had participated in 8 weekly sessions where they have learned a variety of carton crafts techniques, while the control group didn ' $t$ received any intervention. Both groups answered to questionnaires that evaluated their well-being levels. This evaluation was made in the beginning of the first session (pre-test) and in the end of the last session (post-test). The results of a mix ANOVA 2 (group) $\times 2$ (time), with repeated measures in the time factor and independent measures in the group factor had showed that the scores in the affective organizational commitment and the positive affects toward work have been significantly higher, while the emotional exhaustion have been significantly lower at the end of intervention. We concluded that the project was effective in contributing to the user's higher well-being.

Keywords: evaluation, program, well-being, artistic activities.

\section{RESUMEN}

El bienestar laboral tiene recibido destaque en la literatura de psicología organizacional. Se investigó el impacto del proyecto 'Almacén de las Artes' en el bienestar de sus participantes. La muestra de 97 funcionarios de una universidad federal, de ambos sexos, se dividió en un grupo experimental $(\mathrm{N}=48)$ y en un grupo control $(\mathrm{N}=49)$. El grupo experimental participó en ocho sesiones semanales, en las que les ensenaron diversas técnicas de cartonaje y el grupo control no recibió ningún tipo de intervención. Ambos grupos respondieron instrumentos destinados a evaluar sus niveles de bienestar, al inicio de la primera sesión (pre-prueba) y al final de la última sesión (post-prueba). Los análisis de la variancia (ANOVA) mixtas 2 (grupo) X 2 (tiempo), con medidas repetidas en el factor tiempo e independientes en el factor grupos, evidenciaron que los escores de comprometimiento organizacional afectivo y de afectos positivos dirigidos al trabajo fueron mayores al final de la intervención, mientras que los de agotamiento emocional fueron menores al final de la intervención. Se concluye que el proyecto se mostró eficaz por contribuir para mejorar el bienestar de sus participantes.

Palabras clave: evaluación, programas, bienestar, actividades artísticas.

\section{Introdução}

O mundo do trabalho vem passando por transformações decorrentes, sobretudo, da globalização, da maior competitividade e da necessidade de implantação de novas formas de organização no trabalho. Essas mudanças têm impactado as condições de vida do trabalhador e repercutido, direta ou indiretamente, em seu bem-estar, bem como em sua produtividade e na das organizações. Por essa razão, a temática do bem-estar no trabalho tem ocupado lugar de destaque na literatura da área da psicologia organizacional (Fernandes \& Gutierrez, 1988; Ferreira, 2012).

Os estudiosos do bem-estar no trabalho ainda não foram também capazes de obter um consenso acerca de seu significado conceitual e, por essa razão, inúmeras definições podem ser encontradas para o referido construto. De acordo com Van Horn, Taris, Schaufeli e Schreurs (2004), por exemplo, o bem-estar consiste em um conceito 
multidimensional associado à avaliação positiva de características afetivas, motivacionais, comportamentais, cognitivas e psicossomáticas subjacentes ao contexto laboral. Já Siqueira e Padovam (2008) sustentam que o bem-estar no trabalho associa-se a vínculos prazerosos estabelecidos no contexto do trabalho, podendo, assim, ser entendido como um construto psicológico multidimensional, integrado por vínculos afetivos positivos com o trabalho e com a organização. Ele se caracteriza, portanto, pelo predomínio de emoções positivas e pela percepção de que, no seu trabalho, o indivíduo expressa e desenvolve seus potenciais/habilidades e avança no alcance de suas metas de vida.

Cientes das importantes consequências que as condições de trabalho acarretam para o bem-estar dos membros organizacionais, os profissionais de gestão de pessoas têm envidado esforços em direção à adoção de práticas capazes melhorá-las. Dentre essas práticas, merecem destaque os programas de bem-estar no trabalho, que vêm sendo adotados cada vez mais frequentemente pelas organizações. A grande maioria deles, contudo, acaba não sendo objeto de avaliações sistemáticas de sua efetividade (Minayo, 2009). Face a essas considerações, o presente estudo teve como objetivo avaliar o impacto de um projeto chamado de "Armazém da Artes", no bemestar no trabalho de seus participantes.

\section{Dimensões do bem estar no trabalho}

Diferentes modelos têm procurado sistematizar as dimensões do bem-estar no trabalho (Ferreira et al., 2012; Van Horn et al., 2004; Warr, 2005). O modelo de van Horn et al. (2004) utiliza-se de cinco dimensões: afetiva, bem-estar profissional, bem-estar social, bemestar cognitivo e bem-estar psicossomático. A dimensão afetiva do bem-estar no trabalho inclui os afetos positivos dirigidos ao trabalho (sentimentos e emoções), a ausência de exaustão emocional (sentimento de estar livre de sobrecarga emocional) e a satisfação no trabalho (estado íntimo positivo, decorrente do trabalho, que se expressa afetiva e cognitivamente).

O presente estudo adotou o modelo de van Horn et. al (2004), mas trabalhou apenas com a dimensão afetiva do bem-estar no trabalho, em função de eles serem os mais frequentemente adotados nos estudos da área, tendo levado os autores a afirmarem que tal dimensão constitui-se no cerne do construto. Nesse sentido, a satisfação no trabalho, o comprometimento organizacional afetivo, os afetos dirigidos ao trabalho e a falta de exaustão emocional foram utilizados como indicadores da dimensão afetiva do bem-estar no trabalho. 
Para Spector (1997), a satisfação no trabalho está relacionada a como as pessoas se sentem em relação ao seu trabalho, estando associada não apenas ao quanto elas gostam do que realizam, mas, também, a seus sentimentos sobre os diferentes aspectos do mesmo. O comprometimento organizacional afetivo, por sua vez, caracterizase como uma ligação psicológica, de natureza afetiva, com a organização, capaz de fazer o indivíduo adotar os objetivos da organização como se eles fossem seus próprios objetivos (Siqueira \& Gomide Jr., 2004). Os afetos positivos no trabalho podem ser concebidos como respostas emocionais positivas que os indivíduos dirigem a seu ambiente laboral (Ferreira, Silva, Fernandes, \& Almeida, 2008). A exaustão emocional caracteriza-se como uma das dimensões do burnout (síndrome psicológica decorrente do estresse crônico no trabalho) (Tamayo \& Tróccoli, 2002) e refere-se a sentimentos de que todas as forças emocionais e físicas do indivíduo estão sendo exauridas pelo trabalho (Gondim \& Siqueira, 2014).

\section{A avaliação de programas de bem-estar no trabalho}

Um programa caracteriza-se por uma intervenção sistemática, planejada com o objetivo de atingir mudanças na realidade (Cano, 2004). Ele consiste, assim, em um conjunto organizado de atividades destinadas a causar algum tipo de impacto, mediante a adoção de estratégias de intervenção (Royse, Thayer, \& Padgett, 2010). Para além de se desenvolverem os programas, no entanto, torna-se também necessário avaliar se seus objetivos e metas foram atingidos. A avaliação constitui, portanto, parte fundamental dos programas, podendo ser conceituada como uma pesquisa aplicada que faz parte do processo de gerenciamento dos mesmos e tem por objetivo avaliar se eles cumprem ou não as suas metas (Royse et al., 2010).

A análise dos estudos empíricos voltados à avaliação dos programas de bem-estar no trabalho evidencia que a maioria deles tem como objetivo geral a melhoria da saúde física e psicológica dos participantes. Nesse sentido, tais programas utilizam-se com frequência de intervenções (sessões de ginástica laboral, palestras e informações sobre estresse, técnicas de relaxamento etc.) e de comparações entre medidas coletadas no pré e no pós-teste, nos grupos experimental (que recebeu intervenção) e de controle (que não recebeu intervenção), conforme pode ser observado em diferentes estudos (Antunes et al., 2005; Martins \& Michels, 2001; McGrady, Brennan \& Lynch, 2009; Murta \& Tróccoli, 2004; 2009).

Contudo, no levantamento realizado em bases de dados nacionais (SCIELO e PePsic) e estrangeiras (Psychinfo), em julho de 2015, foram localizados somente alguns estudos com foco na promoção do bem-estar mediante 0 uso de atividades artísticas. Um deles 
(Michalos \& Kahlke, 2009) concluiu não ser possível medir o impacto das atividades relativas à arte no bem-estar, em razão do fato de que os instrumentos de medida revelaram-se inapropriados. $O$ estudo de Lipe et al. (2012), no entanto, apontou para um aumento significativo dos índices de bem-estar entre o pré e o pós-teste. No entanto, como a intervenção utilizou ao mesmo tempo atividades musicais e artísticas, não foi possível avaliar o impacto diferencial de cada uma dessas atividades no bem-estar dos participantes. O mesmo ocorreu com a pesquisa de Rego (2008), que contemplou vivências semanais com pinturas, desenhos, música, literatura, colagens, modelagens e criações a partir de sucatas, com resultados positivos no bem-estar e diminuição do estresse ocupacional em profissionais de teleatendimento.

A pesquisa de Castro et al. (2007), por sua vez, encontrou que programas de atividades físicas, de promoção social e cultural (onde se incluía a educação musical, arte e cidadania) e de atividades comemorativas e turismo cultural tinham efeitos positivos no bemestar de adultos de meia-idade e idosos, sem diferenças significativas entre tais atividades. De forma similar, Guedes, Guedes e Almeida (2011) também investigaram o efeito das atividades artísticas sobre os idosos encontrando resultados positivos sobre a autoimagem, autoestima, recuperação biológica, psicológica, social e imunológica dos idosos.

Outros estudos voltados para a área de saúde também relacionam ganhos no bem-estar por meio de vivências e atividades artísticas. São exemplos o estudo de Cabe, Roche, Hegarty e McCann (2013) com pacientes oncológicos que fariam transplante; o de Safar e Press (2011) sobre demência; o de Chancellor, Duncanc e Chatterjee (2014) sobre demência e Alzheimer e a revisão de literatura apresentada por Ehresman (2014) sobre a influência das atividades artísticas no bem-estar de pessoas com Alzheimer.

Justificam-se, dessa forma, estudos adicionais que se mostrem capazes de avaliar mais acuradamente o impacto de programas de intervenção baseados em atividades artísticas no bem-estar no trabalho dos participantes. A realização de estudos dessa natureza poderá contribuir para que cada vez mais os programas de bem-estar do trabalhador demonstrem sua efetividade e passem a ser considerados prioritários e, em consequência, incorporados ao planejamento estratégico das organizações. Apoiando-se em tais considerações, o presente trabalho teve como objetivo geral investigar o impacto do projeto Armazém das Artes no bem-estar no trabalho de seus participantes. 


\section{0 projeto Armazém das Artes}

O projeto avaliado na atual investigação integra o Programa de Qualidade de Vida e Saúde do Servidor de uma universidade federal. O referido programa tem como principal objetivo empreender esforços para melhorar a qualidade de vida dos funcionários da universidade, oferecendo recursos e implementando ações que visam a facilitar o desenvolvimento das habilidades pessoais, proporcionar meios para o enfrentamento de situações limítrofes e apresentar práticas alternativas, complementares e integrativas em saúde, de modo a que os servidores da universidade possam aumentar suas chances de viver mais e melhor. Desde a criação do programa, vários projetos foram implantados, sendo, atualmente, 12 projetos.

Um dos projetos que faz parte do programa é o Armazém das Artes, que tem por objetivo oferecer condições para o desenvolvimento de potencialidades nos indivíduos da comunidade interna da universidade, por meio de oficinas de artesanato, como forma de lhes proporcionar maior bem-estar. A criação do projeto apoiou-se teoricamente no trabalho de Sennet (2009), para quem a competitividade presente na sociedade moderna provoca desigualdades e constitui uma realidade permanente, o que leva à necessidade de as instituições se utilizarem de estratégias capazes de lidar com tal situação. Uma delas seria a descoberta de outras capacitações entre os trabalhadores, que favorecessem sua ligação afetiva positiva com o trabalho e, consequentemente, a vivência de ambientes laborais mais saudáveis. Uma das ferramentas para se atingir tais objetivos é o trabalho manual ou artesanal.

De acordo com Sennet (2009), a habilidade artesanal caracteriza-se como um impulso básico e permanente do ser humano que se manifesta no desejo de fazer um trabalho bem feito. Tais habilidades resgatam, assim, o prazer pelo trabalho, que pode ser generalizado às demais atividades laborais. Utilizando-se, portanto, do trabalho de Sennet (2009) como referencial teórico, o projeto Armazém das Artes destaca a importância de se desenvolverem trabalhos artesanais, não apenas no que diz respeito ao aspecto econômico (como uma possível complementação da renda), mas também como forma de desenvolver a criatividade, proporcionando ao participante a satisfação de descobrir habilidades até então desconhecidas.

O material utilizado no projeto é fornecido pela própria universidade, razão pela qual a participação não implica qualquer custo para os funcionários. Ele se desenvolve em oito encontros semanais de três horas de duração, e a cada oficina são apresentados os materiais a serem utilizados para a confecção dos produtos, como, por exemplo, papelão, tecido, cola etc., além de que a cada encontro, um produto diferente é confeccionado. Cada oficina inicia-se com uma conversa informal sobre a impressão de todos com relação ao trabalho 
executado no encontro anterior, seguida da execução do produto do dia, com uma breve parada para um café após uma hora e meia. Os participantes levam os produtos confeccionados para casa.

Conforme mencionado, o presente estudo procurou avaliar o impacto do projeto Armazém das Artes no bem-estar no trabalho de seus participantes. Fundamentando-se nos estudos empíricos revistos foram, então, formuladas as seguintes hipóteses de pesquisa.

H1- Os escores de satisfação do trabalho dos participantes do projeto Armazém das Artes são significativamente maiores ao final da intervenção quando comparados ao início do projeto.

$\mathrm{H} 2$ - Os escores de comprometimento organizacional afetivo dos participantes do projeto Armazém das Artes são significativamente maiores ao final da intervenção quando comparados ao início do projeto.

H3- Os escores de afetos positivos dos participantes do projeto Armazém das Artes são significativamente maiores ao final da intervenção quando comparados ao início do projeto.

H4- Os escores de exaustão emocional dos participantes do projeto Armazém das Artes são significativamente menores ao final da intervenção quando comparados ao início do projeto.

\section{Método}

\section{Participantes}

A amostra contou com 97 funcionários de uma universidade situada no Estado do Rio de Janeiro. Os participantes distribuíram-se entre o grupo experimental $(N=48)$ e o grupo controle $(N=49)$. No grupo experimental, a maioria era do sexo feminino $(93,9 \%)$. A idade dos participantes desse grupo variou de 27 a 68 anos (média $=49,45$; desvio-padrão $=10,15)$, com a grande maioria apresentando nível superior completo $(85,7 \%)$. No que tange ao estado civil, $52,6 \%$ eram casados, com os demais se distribuindo entre solteiros $(22,6 \%)$, separados $(16,4 \%)$ e viúvos $(8,4 \%)$. Desse total, $67,3 \%$ possuíam filhos (de um a três). A maioria dos participantes $(69,4 \%)$ desse grupo exercia funções administrativas e seu tempo de trabalho na instituição variou de 1 a 47 anos (média $=22,47$; desvio-padrão $=$ $10,54)$, enquanto seu tempo de trabalho total variou de 2 a 43 anos ( média $=24,50$; desvio-padrão $=9,58$ ). Observou-se, ainda, que $67,3 \%$ dos participantes já tinham participado anteriormente de oficinas de artes. Cumpre ressaltar que o fato de o projeto Armazém das Artes desenvolver-se com um número máximo de oito participantes, implicou a realização de sete diferentes edições, sendo que, em cada uma delas, o total de participantes que completou o projeto variou de seis a oito participantes. Tais indivíduos foram 
selecionados por ordem de inscrição, a partir da lista de interessados em participar do projeto, e receberam um código identificador que permitiu o pareamento dos dados do pré e pós-teste de cada participante.

No que se refere ao grupo controle, $77,1 \%$ eram do sexo feminino. A idade dos participantes desse grupo variou de 27 a 61 anos (média $=$ 48,38; desvio-padrão 9,43), sendo que a grande maioria $(79,2 \%)$ apresentou nível superior completo. No que tange ao estado civil, $54,2 \%$ eram casados, $16,7 \%$, solteiros e $29,1 \%$, separados. Desse total, $66,7 \%$ possuíam filhos (de um a quatro). Metade da amostra possuía cargo de supervisão e o tempo de trabalho desses indivíduos variou de 1 a 33 anos (média $=15,85$; desvio-padrão $=12,60$ ), enquanto seu tempo de trabalho total variou de 21 a 37 anos (média $=23,23$; desvio padrão $=10,61$ ). Nesse grupo, $60,4 \%$ dos indivíduos não tinham participado de oficinas de artes anteriormente. Tais indivíduos foram também selecionados por ordem de inscrição, a partir da lista de interessados em participar do projeto, e receberam um código identificador que permitiu o pareamento dos dados do pré e pós-teste de cada participante. Cumpre ressaltar que todos os participantes do grupo de controle, ao final da pesquisa, tiveram oportunidade de participar das oficinas.

\section{Instrumentos}

A satisfação no trabalho foi avaliada com a Escala de Satisfação Geral no Trabalho (Silva \& Ferreira, 2009), que é unifatorial e compõe-se de cinco itens a serem respondidos em escala de seis pontos, variando de discordo fortemente (1) a concordo fortemente (6). Exemplo de item: Sinto-me satisfeito com meu trabalho atual. Seu coeficiente de consistência interna, calculado pelo coeficiente Alfa de Cronbach, foi igual a 0,80 no estudo de validação original. No presente trabalho, o Alfa de Cronbach foi igual a 0,88 no pré-teste, e 0,90 , no pós-teste.

$\mathrm{Na}$ avaliação do comprometimento organizacional, foi utilizada a Escala de Comprometimento Afetivo de Meyer e Allen (1991), em versão adaptada para o Brasil por Ferreira et al. (2002). A referida escala consta de seis itens, a serem respondidos em escalas de seis pontos, variando de discordo totalmente (1) a concordo totalmente (6), de acordo com os sentimentos manifestados pelo respondente em relação à sua organização. Exemplo de item: Sinto-me de fato pertencendo a minha organização. No processo de validação original, a escala obteve um índice de consistência interna igual a 0,85, calculado pelo Alfa de Cronbach. No presente trabalho, o Alfa de Cronbach foi igual a 0,86 no pré-teste, e a 0,88 , no pós-teste.

Os afetos positivos dirigidos ao trabalho foram avaliados pela versão reduzida da sub-escala de Afetos Positivos, pertencentes à Escala de 
Afetos no Trabalho (ESAFE), de autoria de Ferreira et al. (2008), a qual consta de dez itens, a serem respondidos em escalas de cinco pontos, variando de nunca (1) a sempre (5). Exemplo de item: Sou capaz de encarar os problemas. O índice de consistência interna, no estudo original, foi igual a 0,93 . No presente trabalho, o Alfa de Cronbach foi igual a 0,92 no pré-teste, e a 0,93, no pós-teste.

A exaustão emocional foi avaliada pela escala destinada a esse fim no Inventário de Burnout de Malasch - General Survey (MBI-GI), desenvolvido por Schaufeli, Leiter, Malash e Jackson (1996), especificamente para profissionais sem contato direto com clientes e público em geral, e validado em amostras brasileiras por Silva Jr. (2005). Tal questionário é constituído de cinco itens, a serem respondidos em escalas de cinco pontos, variando de nunca (1) a diariamente (5). Exemplo de item: Sinto-me consumido pelo meu trabalho. O coeficiente de precisão dessa escala, no estudo original de adaptação, foi igual a 0,80 . No presente trabalho, o Alfa de Cronbach foi igual a 0,84 no pré-teste, e a 0,67 , no pós-teste.

\section{Procedimentos}

O projeto foi inicialmente submetido à avaliação e aprovação do Comitê de Ética em Pesquisa da instituição dos autores. Em seguida, realizaram-se as oito edições do projeto Armazém das Artes no grupo experimental, sendo cada uma composta de oito encontros semanais sucessivos, perfazendo um período total de 2 meses em cada edição do projeto. No primeiro dia, após a apresentação dos objetivos do projeto, a instrutora distribuiu os instrumentos de coleta de dados e solicitou o seu preenchimento, explicando que ele era voluntário, muito embora todos os participantes tenham sido unânimes em concordar com o preenchimento e em serem identificados por um código. Posteriormente, foram realizadas as atividades previstas para o primeiro encontro, o mesmo ocorrendo nas demais seções. Nesse sentido, a cada oficina eram apresentados os materiais a serem utilizados (papelão Horle, papel collor plus, papel fantasia, papéis artesanais, cola, régua de metal, lápis, borracha, faca olfa, dobradeira, espátulas, placa de corte, tesouras, fechos metálicos, tecidos de algodão, elástico, fita crepe, papel chamex e caderno para anotações) e confeccionados produtos de crescente complexidade, a saber: caixa quadrada ( 10 encontro); bloco brochura ( 2 o encontro); caixa para chá ( $3 \circ$ encontro); risque e rabisque ( 4 ㅇ encontro); porta documentos e porta cartão ( 5 o encontro); caixa de costura ( 6 o e 70 encontros); porta recados ( 8 o encontro). No oitavo e último encontro, após a realização das atividades previstas, os participantes foram novamente solicitados a preencher os mesmos instrumentos respondidos ao início da intervenção. Vale ressaltar que todos os 
encontros e edições do projeto foram conduzidas pela mesma instrutora.

No grupo controle, os trabalhadores foram contatados e voluntariamente concordaram em participar da pesquisa, tendo respondido, nas mesmas ocasiões, aos instrumentos aplicados aos participantes nas sessões inicial e final do grupo experimental. Eles também responderam e devolveram os questionários imediatamente após o seu preenchimento. A todos os participantes, de ambos os grupos, foi assegurado o sigilo acerca das informações coletadas.

\section{Resultados}

Para verificar se os participantes dos grupos experimental e de controle apresentavam diferenças significativas em suas características sociodemográficas, foram realizados testes de quiquadrado para as comparações entre os grupos, em função do sexo, estado civil, escolaridade, ter ou não filhos, salários e nível organizacional, e testes $t$ para amostras independentes, nas comparações entre os grupos, nas variáveis idade, tempo atual de trabalho e tempo total de trabalho. Os dados obtidos demonstraram haver diferenças significativas entre os dois grupos em função do sexo $\left(X^{2}=5,53 ; p<0,05\right)$; do nível organizacional $\left(X^{2}=33,62 ; p<\right.$ $0,01)$ e do tempo de trabalho atual $(t=2,77 ; p<0,01)$.

As médias dos escores obtidos nas diversas medidas do estudo, nos grupos experimental e de controle, bem como na amostra total, no pré e no pós-teste, são apresentadas na Tabela 1. Para se verificar a existência de diferenças entre as diversas medidas de bem-estar utilizadas no pré e pós-teste, foi realizada uma análise da variância (ANOVA) mista 2 (grupo) X 2 (tempo), com medidas repetidas (intrasujeitos) no fator tempo e com medidas independentes no fator grupo (entre sujeitos), para cada uma das variáveis dependentes do estudo, após o exame dos pressupostos para esse tipo de teste (Tabela 2). Nesse sentido, verificou-se inicialmente que as variáveis dependentes eram normalmente distribuídas, que as variâncias dos grupos eram homogêneas e que não havia a presença de outliers (escores extremos) em nenhuma das distribuições, o que evidenciou a pertinência da ANOVA ser adotada na comparação entre as médias dos grupos experimental e de controle. 
Tabela 1.

Estatísticas descritivas obtidas para as diferentes escalas do estudo

\begin{tabular}{lcccccc}
\hline \multirow{2}{*}{ Medidas } & \multicolumn{2}{c}{$\begin{array}{c}\text { Grupo } \\
\text { experimental } \\
\mathrm{N}=49\end{array}$} & \multicolumn{2}{c}{$\begin{array}{c}\text { Grupo controle } \\
\mathrm{N}=48\end{array}$} & \multicolumn{2}{c}{$\begin{array}{c}\text { Amostra total } \\
\mathrm{N}=97\end{array}$} \\
\cline { 2 - 8 } & $\mathrm{M}$ & $\mathrm{DP}$ & $\mathrm{M}$ & $\mathrm{DP}$ & $\mathrm{M}$ & $\mathrm{DP}$ \\
\hline Satisfação no trabalho antes & 3,73 & 0,93 & 3,37 & 0,92 & 3,55 & 0,93 \\
Satisfação no trabalho depois & 3,85 & 1,00 & 3,21 & 0,92 & 3,61 & 1,00 \\
Comprometimento org. antes & 3,56 & 0,80 & 3,21 & 0,83 & 3,38 & 0,83 \\
Comprometimento org. depois & 3,78 & 0,80 & 3,10 & 0,83 & 3,43 & 0,87 \\
Afetos positivos antes & 3,60 & 0,75 & 3,58 & 0,70 & 3,60 & 0,71 \\
Afetos positivos depois & 3,85 & 0,70 & 3,45 & 0,70 & 3,65 & 0,70 \\
Exaustão emocional antes & 2,53 & 0,94 & 2,45 & 1,06 & 2,50 & 1,00 \\
Exaustão emocional depois & 2,23 & 0,92 & 2,65 & 1,41 & 2,42 & 1,20 \\
\hline
\end{tabular}

Tabela 2.

Resultados da ANOVA para as diferentes variáveis dependentes do estudo

\begin{tabular}{lrrrrr}
\hline Fonte de variância & $\begin{array}{l}\text { Soma dos } \\
\text { quadrados }\end{array}$ & df & $\begin{array}{l}\text { Quadrados da } \\
\text { média }\end{array}$ & $F$ & $p$ \\
\hline Satisfação no trabalho & 8,85 & 1 & 8,85 & 5,23 & $<0,025$ \\
Grupo & 160,75 & 95 & 1,69 & & \\
Erro (Grupo) & 0,17 & 1 & 0,17 & 1,72 & 0,193 \\
Tempo & 9,35 & 95 & 0,10 & & \\
Erro (Tempo) & 0,22 & 1 & 0,22 & 2,24 & 0,138 \\
Grupo x Tempo & & & & & \\
Comprometimento & 12,81 & 1 & 12,81 & 10,23 & $<0,01$ \\
Grupo & 118,90 & 95 & 1,25 & & \\
Erro Grupo & 0,14 & 1 & 0,14 & 1,75 & 0,189 \\
Tempo & 7,46 & 95 & 0,08 & & \\
Erro (Tempo) & 1,31 & 1 & 1,31 & 16,70 & $<0,001$ \\
Grupo x Tempo & & & & & \\
Afetos & 2,20 & 1 & 2,20 & 2,64 & 0,107 \\
Grupo & 14,36 & 95 & 0,15 & & \\
Erro (Grupo) & 0,20 & 1 & 0,20 & 1,20 & 0,278 \\
Tempo & 14,36 & 95 & 0,15 & & \\
Erro (Tempo) & 1,71 & 1 & 1,71 & 11,33 & $<0,001$ \\
Grupo x Tempo & & & & & \\
& 1,14 & 1 & 1,14 & 0,54 & 0,462 \\
Exaustão & 30,72 & 95 & 0,32 & & \\
Grupo & 0,19 & 1 & 0,19 & 0,60 & 0,440 \\
Erro (Grupo) & 30,72 & 95 & 0,32 & & \\
Tempo & 2,66 & 1 & 2,66 & 8,23 & $<0,01$ \\
Erro (Tempo) & & & & & \\
Grupo x Tempo & & & & & \\
\hline
\end{tabular}


No que diz respeito à satisfação com o trabalho, a interação entre grupo e tempo não foi significativa, levando assim à rejeição da Hipótese 1, segundo a qual os escores de satisfação do trabalho dos participantes do projeto Armazém das Artes seriam significativamente maiores ao final da intervenção, quando comparados aos obtidos ao início do projeto. Atendo-se aos escores de comprometimento organizacional afetivo, foi observado que a interação entre grupo e tempo foi significativa. $O$ teste $t$ realizado posteriormente evidenciou que a diferença entre o pré e o pós-teste no grupo controle foi significativa $(t(47)=-2,45 ; p<0,05)$, porém no sentido de os escores do pós-teste serem significativamente menores do que os do pré-teste. No grupo experimental, entretanto, conforme seria de se esperar, as médias do pós-teste foram significativamente maiores do que as médias do pré-teste $(t(48)=3,44 ; p<0,001)$, o que confirma a Hipótese 2 da pesquisa, que previa o aumento dos escores de comprometimento organizacional afetivo dos participantes do projeto ao final da intervenção, quando comparados aos escores obtidos ao início do projeto (Figura 1).

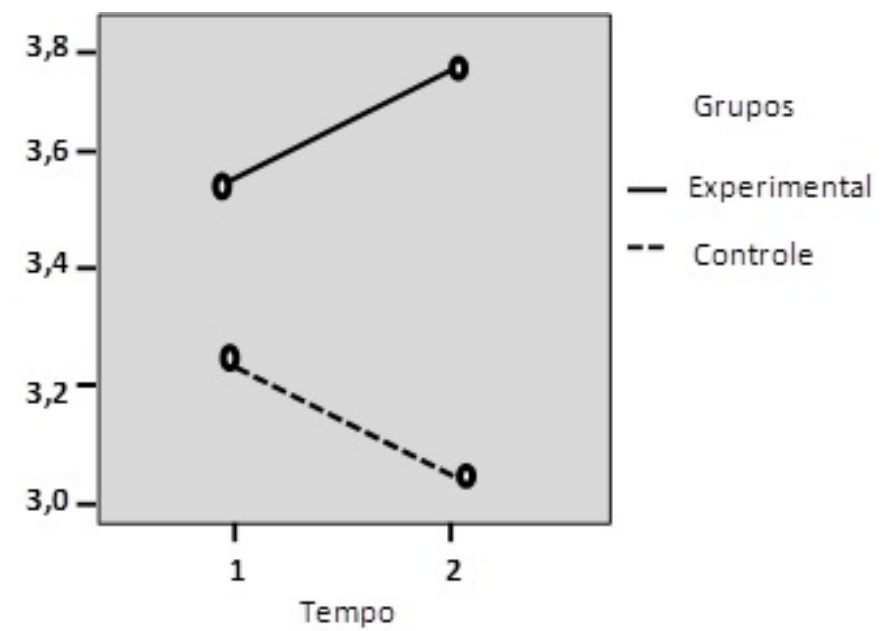

Figura 1. Interação entre grupo e tempo na escala de comprometimento organizacional afetivo.

Para os afetos positivos dirigidos ao trabalho, foi observado que a interação entre grupo e tempo foi significativa. $O$ teste $t$ realizado na sequência demonstrou que a diferença entre o pré e o pós-teste no grupo controle foi significativa $(t(47)=-2,90 p<0,05)$, porém no sentido de os escores do pós-teste serem significativamente menores do que os escores do pré-teste. No grupo experimental, contudo, conforme seria de se esperar, as médias do pós-teste foram significativamente maiores do que as médias do pré-teste $(t(48)=-$ $2,44 ; p<0,005)$, o que confirma a Hipótese 3 da pesquisa, segundo a qual os escores de afetos positivos dos participantes do projeto seriam significativamente maiores ao final da intervenção, quando comparados aos escores obtidos no início do projeto (Figura 2). 


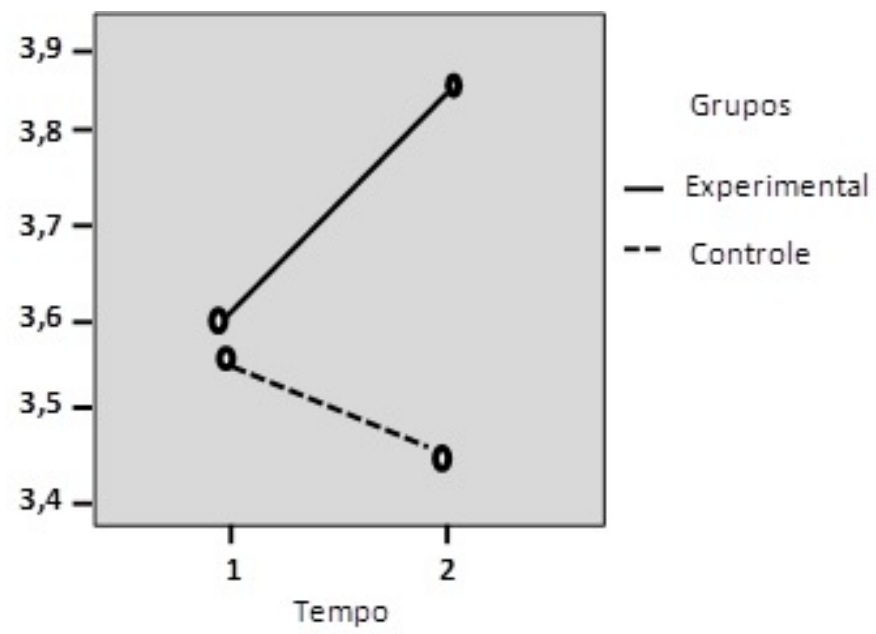

Figura 2. Interação entre, grupo e tempo na escala de afetos positivos dirigidos ao trabalho.

No caso da exaustão emocional, a interação entre grupo e tempo foi significativa. O teste $t$ realizado posteriormente evidenciou que a diferença entre o pré e o pós-teste, no grupo controle, não foi significativa. No entanto, no grupo experimental, como seria de se esperar, as médias do pós-teste foram significativamente menores do que as médias do pré-teste $(t(48)=-3,25 p<0,01)$, o que confirma a Hipótese 4 da pesquisa, que previa a diminuição dos escores de exaustão emocional dos participantes do projeto ao final da intervenção, quando comparados aos escores obtidos ao início do projeto (Figura 3).

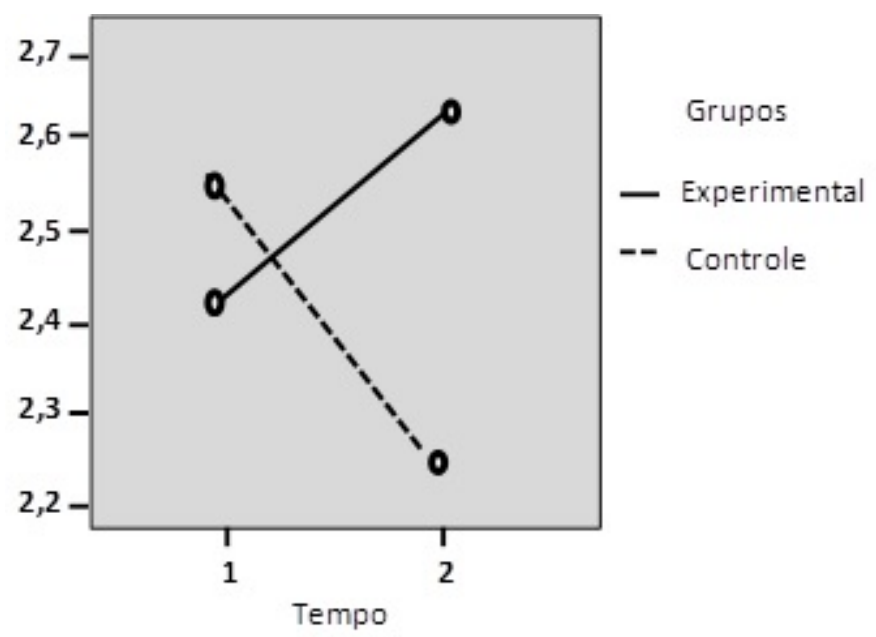

Figura 3. Interação entre, grupo e tempo na escala de exaustão emocional.

\section{Discussão}

A presente pesquisa teve como objetivo investigar o impacto do projeto Armazém das Artes no bem-estar de seus participantes, 
adotando, para tal, quatro indicadores desse construto: satisfação no trabalho, comprometimento organizacional afetivo, afetos positivos dirigidos ao trabalho e exaustão emocional. A Hipótese 1 previa que os escores de satisfação no trabalho dos participantes do projeto Armazém das Artes seriam, ao final da intervenção, significativamente maiores que aqueles do início do projeto. Os dados obtidos revelaram, porém, que os participantes não apresentaram índices maiores de satisfação no trabalho após terem participado do projeto, o que impediu a confirmação dessa hipótese.

A Hipótese 2 pressupunha que os escores de comprometimento organizacional afetivo dos participantes do projeto Armazém das Artes seriam significativamente maiores ao final da intervenção, quando comparados ao início do projeto, o que se confirmou totalmente. O comprometimento organizacional afetivo configura uma relação que se define pelo desejo de permanecer na organização, em função dos vínculos afetivos que os indivíduos mantêm com a mesma (Siqueira \& Padovam, 2008). Nesse sentido, o trabalhador comprometido acredita e internaliza os valores da organização, identifica-se com seus objetivos e se envolve com os papéis de trabalho, desempenhando-os de forma a facilitar a consecução dos objetivos organizacionais (Siqueira, 2005). Os resultados obtidos evidenciaram, portanto, que os participantes do projeto apresentaram maiores vínculos afetivos com sua organização empregadora após participar do projeto, em que pese o fato de que, em razão de serem servidores públicos, dificilmente tomariam decisões de deixar a organização, caso seus vínculos afetivos com ela se deteriorassem.

De acordo com a Hipótese 3, os escores de afetos positivos dos participantes do projeto Armazém das Artes seriam significativamente maiores ao final da intervenção, quando comparados ao início do projeto, o que de fato foi confirmado. Os afetos positivos podem ser concebidos como sentimentos transitórios, de caráter hedônico, que se manifestam em reações de entusiasmo, otimismo etc. (Albuquerque \& Tróccoli, 2008). Nesse sentido, os participantes do Projeto demonstraram que o trabalho por eles desenvolvido durante a intervenção deixou-os mais otimistas e entusiasmados com seu trabalho.

Por fim, a Hipótese 4 supunha que os escores de exaustão emocional dos participantes do Projeto Armazém das Artes seriam significativamente menores ao final da intervenção, quando comparados ao início do Projeto, o que se confirmou. A exaustão ou esgotamento profissional diz respeito a sentimentos de que todas as forças emocionais e físicas do indivíduo estão sendo exauridas pelo trabalho (Tamayo \& Tróccoli, 2002). Constatou-se, portanto, que após a participação no projeto, os participantes passaram a se sentir menos esgotados ao final do dia de trabalho, com mais disposição 
para levantar de manhã e menos estressados no trabalho e no relacionamento com as pessoas.

Tomados em conjunto, os dados ora obtidos para as medidas de comprometimento organizacional, afetos positivos no trabalho e exaustão emocional convergem com os achados anteriores de Petterson e Arnetz (1998), Martins e Michels (2001), Antunes et al. (2005), Murta e Tróccoli (2004; 2009) e McGrady et al. (2009), nos quais também foram adotadas intervenções de diferentes modalidades. Esses programas, porém, recorreram à utilização de atividades físicas, relaxamento, discussões e workshops. Tais dados podem ser vistos ainda como uma extensão da investigação anterior de Lipe et al. (2002), que revelou evidências sobre a efetividade de intervenções artísticas no bem-estar, ao demonstrarem que o projeto Armazém das Artes mostrou-se capaz de contribuir para a melhoria do bem-estar no trabalho de seus participantes. Tal resultado também confirma os achados de Rego (2008), que identificou redução do estresse e aumento do bem-estar no trabalho de atendentes de telemarketing que participaram de programas de atividades artísticas.

Cumpre discutir, por fim, os motivos pelos quais o programa não surtiu efeito com a variável satisfação no trabalho. O referido construto caracteriza-se por atitudes que possuem uma dimensão afetiva e outra cognitiva (Siqueira \& Kurcgant, 2012). Considerandose que as atividades artísticas se apresentam mais associadas às emoções (Sennett, 2009), é provável, então, que o projeto Armazém das Artes tenha impactado apenas as variáveis de natureza mais afetiva, como é o caso dos afetos, da exaustão emocional e do comprometimento afetivo, deixando assim de afetar a satisfação com o trabalho, devido à sua natureza mais cognitiva.

Em síntese, os atuais achados podem ser tomados como evidências de que a aprendizagem de habilidades manuais por meio de intervenções artísticas sistemáticas e dirigidas reveste-se da capacidade potencial de sensibilizar e motivar os participantes a desenvolverem atitudes mais positivas em relação a seu trabalho e a sua organização empregadora. Consequentemente, seus índices de bem-estar no trabalho tornam-se também mais favoráveis.

Tais resultados permitem a elaboração de algumas sugestões de estratégias que podem ser futuramente implementadas com 0 objetivo de contribuir para a melhoria do bem-estar no trabalho dos funcionários da universidade. Nesse sentido, a ampla divulgação dos resultados ora obtidos no interior da instituição pesquisada poderá fortalecer a cultura de promoção de saúde do trabalhador e levar a um incremento da taxa de participação dos funcionários em futuras edições do projeto Armazém das Artes, bem como em outros programas que venham a ser lançados com foco no bem-estar do trabalhador. 
Em relação a uma agenda para futuras pesquisas, sugere-se a análise da efetividade do projeto Armazém das Artes por meio de outras medidas, como, por exemplo, indicadores de saúde física, ou mediante levantamentos de dados qualitativos, que possam contribuir para o aprofundamento do conhecimento acerca dos efeitos do projeto. Adicionalmente também seria interessante que outros projetos inseridos no Programa de Qualidade de Vida do Servidor fossem submetidos a avaliações de efetividade, como ocorreu com o projeto Armazém das Artes.

Cumpre ressaltar, por fim, as limitações da presente pesquisa. Nesse sentido, o fato de ela ter sido quase-experimental e de sua amostra ter sido composta tão somente por funcionários estáveis de uma universidade pública limita as possibilidades de generalização. Outrossim, o fato de algumas diferenças entre as médias terem sido pequenas em termos absolutos, embora estatisticamente significativas, constitui outra limitação do estudo e sinaliza a necessidade de realização de estudos futuros sobre o projeto em tela, com os mesmos instrumentos de avaliação do bem-estar aqui adotados, bem como com outros indicadores do referido construto, de modo a se verificar se os achados ora obtidos podem ser replicados. De todo modo, ela se reveste de importância teórica e prática, por representar um primeiro passo em direção à avaliação mais sistemática da efetividade de programas de intervenção ancorados em atividades artísticas e destinados a aumentar o bemestar no contexto laboral, área na qual os esforços empíricos ainda têm sido incipientes.

\section{Referências}

Albuquerque, A. S., \& Tróccoli, B. T. (2004). Desenvolvimento de uma escala de bem-estar subjetivo. Psicologia: Teoria e Pesquisa, 20, 153-164. http://www.scielo.br/pdf/ptp/v20n2/a08v20n2

Antunes, H. K. M., Stella, S. G., Santos, R. F., Bueno, O. F. A., \& Mello, M. T. (2005). Depression, anxiety and quality of life scores in seniors after an endurance exercise program. Revista Brasileira de Psiquiatria, 27, 266-271. doi: 10.1590/S151644462005000400003

Cabe, C., Roche, D., Hegarty, F. M. \& McCann, S. (2013). 'Open window': A randomized trial of the effect of new media art using a virtual window on quality of life in patients' experiencing stem cell transplantation. Psycho-Oncology, 22, 330-337. doi: 10.1002/pon. 2093.

Cano, I. (2004). Introdução a avaliação de programas sociais. São Paulo: Ed. FGV. 
Castro, P. C., Tahara, N., Rebelatto, J. R., Driusso, P., Aveiro, M. C., \& Oishi, J. (2007). Influência da universidade aberta da terceira idade (UATI) e do programa de revitalização (REVT) sobre a qualidade de vida de adultos de meia-idade e idosos. Brazilian Journal of Physical Therapy, 11, 461-467. doi: 10.1590/S141335552007000600007.

Chancellor, B., Duncanc, A., \& Chatterjee, A. (2014). Art therapy for Alzheimer's disease and other dementias. Journal of Alzheimer's Disease 39, 1-11. doi: 10.3233/J AD-131295.

Ehresman, C. (2014) From rendering to remembering: Art therapy for people with Alzheimer's disease. International Journal of Art Therapy, 19, 43-45. doi: 10.1080/17454832.2013.819023.

Fernandes, E. C., \& Gutierrez, L. H. (1988). Qualidade de vida no trabalho (QVT): Uma experiência brasileira. Revista de Administração da USP, 23, 29-38.

Ferreira, M. C. (2012). Qualidade de vida no trabalho: Uma abordagem centrada no olhar dos trabalhadores. Brasília: Paralelo 15.

Ferreira, M. C., Assmar, E. M. L., Souto, S. O. Braga, L. L., Rocha, P. M., \& Pacheco, G. L. (2002). Validação de um instrumento de comprometimento organizacional. Anais do I Congresso Brasileiro Psicologia Ciência e Profissão. Brasília: Conselho Federal de Psicologia. http://anais.cbp.cfp.org.br/congre/

Ferreira, M. C., Silva, A. P. C., Fernandes, H. A., \& Almeida, S. P. (2008). Desenvolvimento e validação de escala de afetos no trabalho. Avaliação Psicológica, 7, 143-150. http: // pepsic. bvsalud.org/scielo. php?pid=S1677$04712008000200005 \&$ script $=$ sci_arttext

Ferreira, M. C., Souza, M. A., \& Silva, A. C. (2012). Qualidade de vida e bem-estar no trabalho: Principais tendências e perspectivas teóricas. In M. C. Ferreira, \& H. Mendonça (Eds.), Saúde e bemestar no trabalho: Dimensões individuais e culturais (pp. 11-28). São Paulo: Casa do Psicólogo.

Gondim, S., \& Siqueira, M. (2014). Emoções e afetos no trabalho. In J. C. Zanelli, J. E. Borges-Andrade, \& A. V. B. Bastos (Eds.). Psicologia, organizações e trabalho no Brasil (pp. 285-315). Porto Alegre: Artmed.

Guedes, M. H. M., Guedes, H. M., \& Almeida, M. E. F. (2011). Efeito da prática de trabalhos manuais sobre a autoimagem de idosos. Revista Brasileira de Geriatria e Gerontologia, 14, 731-742. doi: 10.1590/S1809-98232011000400012.

Lipe, A. W., Ward, K. C., Watson, A. T., Manley, K., Keen, R., Kelly, J., \& Clemmer, J. (2012). The effects of an arts intervention program in a community mental health setting: A collaborative approach. The Arts in Psychotherapy, 39, 25-30. doi: 10.1016/j.aip.2011.11.002 
Martins, C. O., \& Michels, G. (2001). Saúde x lucro: Quem ganha com um programa de promoção da saúde do trabalhador? Revista Brasileira de Cineantropometria \& Desempenho Humano, 3, 95101.

https://periodicos.ufsc. br/index. php/rbcdh/article/viewFile/400 $1 / 3398$

McGrady, A., Brennan, J., \& Lynch, D. (2009). Effects of wellness programs in family medicine. Applied Psychophysiological Biofeedback, 34, 121-126. doi: 10.1007/s10484-009-9084-3

Meyer, J. P., \& Allen, N. J. (1991). A three-component conceptualization of organizational commitment. Human Resources Management Review, 1, 61-89. doi: 10.1016/10534822(91) $90011-Z$

Michalos, A. C., \& Kahlke, P. M. (2009). Impact of arts - related activities on the perceived quality of life. Social Indicators Research, 89, 193-258. doi: 10.1007/sll205-007-9236-x

Minayo, M. C. S. (2009). Construção de indicadores qualitativos para avaliação de mudanças. Revista Brasileira de Educação Médica, 33, 83-91. http://www.scielo.br/pdf/rbem/v33s1/a09v33s1.pdf

Murta, S. G., \& Tróccoli, B. T. (2004). Avaliação de intervenção em estresse ocupacional. Psicologia: Teoria e Pesquisa, 20, 39-47. doi: 10.1590/S0102-37722004000100006

Murta, S. G., \& Tróccoli, B. T. (2009). Intervenções psicoeducativas para manejo de estresse ocupacional: Um estudo comparativo. Revista Brasileira de Terapia Comportamental e Cognitiva, 11, 25-42.

http://www.usp. br/rbtcc/index. php/RBTCC/article/view/382/28 2

Petterson, I. L., \& Arnetz, B. B. (1998). Psychosocial stressors and well-being in health care workers. The impact of an intervention program. Social Science \& Medicine, 47, 1763-1772. http://www.ncbi.nlm.nih.gov/pubmed/9877346

Rego, R. M. (2008). Expressão artística e teleatendimento: Perspectivas para a melhoria de qualidade de vida no trabalho. Psicologia: Ciência e Profissão, 28, 200-209. doi: 10.1590/S1414-98932008000100015.

Royse, D., Thayer, B. A., \& Padgett, D. K. (2010). Program evaluation: An introduction (5th ed.). Belmont, CA: Brooks-Cole.

Safar, L. T., \& Press, D. Z. (2011). Art and the brain: Effects of dementia on art production in art therapy. Art Therapy: Journal of the American Art Therapy Association, 28, 96-103. doi: 10.1080/07421656.2011.599734

Schaufeli, W. B., Leiter, M. P., Maslach, C., \& Jackson, S. E. (1996). The MBI-General Survey. In C. Maslach, S. E. Jackson, \& M. P. Leiter. Maslach Burnout Inventory manual (3rd ed.). Palo Alto, CA: Consulting Psychologists Press. 
Sennett, R. (2009). O artífice. Rio de Janeiro: Record.

Silva Júnior, D. I. (2005). A relação entre estressores ambientais e burnout em trabalhadores off-shore. Dissertação de Mestrado, Universidade Gama Filho, Rio de Janeiro, Brasil.

Silva, A. P. C., \& Ferreira, M. C. (2009). Escala de satisfação geral no trabalho. Anais do IV Congresso Brasileiro de Avaliação Psicológica, Campinas: IBAP. http://www.ibapnet. org.br/congresso2009/index.html

Siqueira, M. M. M. (2005). Esquema mental de reciprocidade e influências sobre afetividade no trabalho. Estudos de Psicologia, 10, 83-93.

Siqueira, M. M. M., \& Gomide Jr., S. (2004). Vínculos do indivíduo com o trabalho e com a organização. In J. C. Zanelli, J. E. Borges-Andrade, \& A. V. B. Bastos (Eds.), Psicologia, organizações e trabalho no Brasil (pp. 300-328). Porto Alegre: Artmed.

Siqueira, M. M. M., \& Padovam, V. A. R. (2008). Bases teóricas de bem-estar subjetivo, bem-estar psicológico e bem-estar no trabalho. Psicologia: Teoria e Pesquisa, 24, 201-209. doi: 10.1590/S0102-37722008000200010

Siqueira, V. T. A., \& Kurcgant, P. (2012). Satisfação no trabalho: Indicador de qualidade no gerenciamento de recursos humanos em enfermagem. Revista Escola de Enfermagem, 46, 151-157. http://www.scielo.br/pdf/reeusp/v46n1/v46n1a21.pdf

Tamayo, M. R.,\&Tróccoli, B. T. (2002). Exaustão emocional: Relações com a percepção de suporte organizacional e com as estratégias de coping no trabalho. Estudos de Psicologia, 7, 3746. doi: 10.1590/S1413-294X2002000100005

Van Horn, J. E., Taris, T. W., Schaufeli, W. B., \& Schreurs, P. J. G. (2004). The structure of occupational well-being: A study among Dutch teachers. Journal of Occupational and Organizational Psychology, 77, 365-375. doi: 10.1348/0963179041752718.

Warr, P. (2005). Work, well-being, and mental health. In E. K. Kelloway, \& M. R. Frone (Eds.), Handbook of work stress (pp. 547-573). London: Sage Publications.

\section{Endereço para correspondência \\ Luciana Nacif Pinheiro}

Universidade Salgado de Oliveira - UNIVERSO

Rua Marechal Deodoro, 217, Centro, CEP 24030-060, Niterói - RJ, Brasil

Endereço eletrônico: nacifluciana@yahoo.com.br

Maria Cristina Ferreira

Universidade Salgado de Oliveira - UNIVERSO

Rua Marechal Deodoro, 217, Centro, CEP 24030-060, Niterói - RJ, Brasil

Endereço eletrônico: mcris@centroin.com.br 


\section{Luciana Mourão}

Universidade Salgado de Oliveira - UNIVERSO

Rua Marechal Deodoro, 217, Centro, CEP 24030-060, Niterói - RJ, Brasil

Endereço eletrônico: mourao.luciana@gmail.com

Recebido em: 10/09/2015

Reformulado em: 21/12/2016

Aceito para publicação em: 29/12/2016

\section{Notas}

* Mestre em Psicologia pela Universidade Salgado de Oliveira - Niterói/RJ - Brasil e funcionária da Universidade Federal Fluminense.

** Doutora em Psicologia. Professora do Programa de Pós-graduação da Universidade Salgado de Oliveira - Niterói/RJ - Brasil.

*** Doutora em Psicologia. Professora do Programa de Pós-graduação da Universidade Salgado de Oliveira - Niterói/RJ - Brasil. 